\section{Cutaneous Amyloidosis Associated with Amyopathic Dermatomyositis}

\section{To the Editor:}

Localized cutaneous amyloidoses can be divided into primary cutaneous amyloidosis and secondary cutaneous amyloidosis. Primary cutaneous amyloidosis is defined as deposition of amyloid in the skin in the absence of systemic involvement. Secondary cutaneous amyloidosis is associated with secondary amyloid deposition and is accompanied by skin neoplasms such as seborrheic keratosis, Bowen's disease, basal cell carcinoma, porokeratosis, and trichoepithelioma. The cutaneous amyloid, so-called "amyloid-K," is reported to be derived from cytokeratins ${ }^{1-3}$. We describe a case of amyopathic dermatomyositis (ADM) associated with cutaneous amyloidosis.

A 61-year-old woman with dyspnea and general fatigue was referred to our hospital. Two weeks before, she had developed fatigue and dyspnea. On admission, examination showed Raynaud's phenomenon, Gottron's sign, and periungual erythema (Figure 1), and poikiloderma on the trunk, without myalgia or muscle weakness. Skin biopsy of the right fourth finger showed positive staining with hematoxylin-eosin (Figure 2A; arrow) and positive staining with congo red in the papillary dermis (Figure 2B), indicating cutaneous amyloidosis that developed into ADM. The congo red-positive area was confirmed by green birefringence. In addition, immunohistochemical staining of the amyloid deposited lesion resulted in positive staining with anti-amyloid $\mathrm{P}$ antibody (Figure 2C; arrow) and anti-cytokeratin 5 antibody (Figure 2D; arrow), but negative staining with anti-amyloid A antibody, anti-kappa-chain antibody, and anti-lambda-chain antibody (not shown). Laboratory findings showed normal peripheral blood cell count and normal myogenic enzyme level, such as CPK (45 IU/l) and myoglobin $(33 \mathrm{ng} / \mathrm{ml}$; normal $<98 \mathrm{ng} / \mathrm{ml})$. ELISA assay for autoantibodies

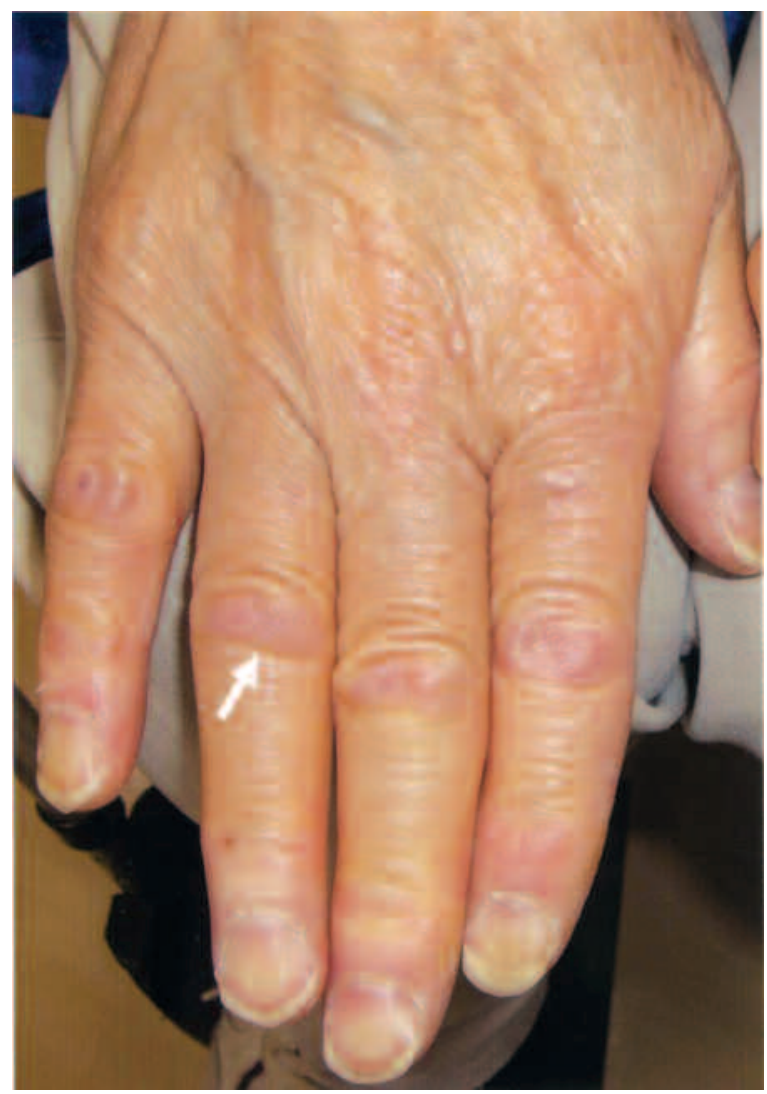

Figure 1. Macroscopic findings of the hands. A. Gottron's sign was noted on the proximal interphalangeal joint. Periungual erythema and capillary loops in proximal nail-folds were also revealed. showed positive levels of anti Jo-1 antibody $(193.5 \mathrm{U} / \mathrm{ml} ;$ normal $<9 \mathrm{U} / \mathrm{ml})$ and anti SSA/Ro antibody. KL-6, a lung fibrosis marker, was $700 \mathrm{U} / \mathrm{ml}$. Serum amyloid A was normal. Chest computed tomography revealed interstitial pneumonia. She was diagnosed with ADM associated with interstitial pneumonia and cutaneous amyloidosis.

Intravenous methylprednisolone pulse therapy $(500 \mathrm{mg}$ daily for 3 days) was started, followed by oral prednisolone $40 \mathrm{mg}$ daily. Intravenous cyclophosphamide $500 \mathrm{mg}$ monthly was added. Three months after starting these therapies, her colors of Gottron's sign and periungual erythema gradually reduced and had disappeared after 6 months.

It is uncommon that cutaneous amyloidosis occurs in patients with $\mathrm{DM}^{4-6}$. A search of the PubMed database identified only 3 cases of patients with DM associated with cutaneous amyloidosis ${ }^{4-6}$. Previous reports described that the duration from the onset of eruption to the diagnosis of cutaneous amyloidosis by skin biopsy was approximately 10 years ${ }^{4-6}$. In patients with DM, cutaneous amyloidosis might be based on chronic inflammation. In our case and in previous reports complications including interstitial pneumonia, Raynaud's phenomenon, periungual erythema, and Gottron's sign have occurred. Because of positive anti-cytokeratin 5 antibody staining, we suggest that in patients with DM the cutaneous amyloidosis might derive mainly from cytokeratin 5 of basal keratinocytes as well as Bowen's disease, basal cell carcinoma, and actinic keratosis ${ }^{2,3}$.

In patients with DM, the association of cutaneous amyloidosis and DM might be more frequent than we thought. Thus, testing of patients with DM for cutaneous amyloid could be worthwhile.

YOSHINORI TANIGUCHI, MD; TARO HORINO, MD, PhD; and YOSHIO TERADA, MD, PhD, Department of Endocrinology, Metabolism and Nephrology, Kochi Medical School, Kochi, Japan. Address reprint requests to Dr. Y. Taniguchi, Department of Endocrinology, Metabolism and Nephrology, Kochi Medical School, Kohasu, Oko-cho, Nankoku, Kochi 783-8505, Japan. E-mail: jm-yoshii@kochi-u.ac.jp

\section{REFERENCES}

1. Kumakiri M, Hashimoto K. Histogenesis of primary localized cutaneous amyloidosis: Sequential change of epidermal keratinocytes to amyloid via filamentous degeneration. J Invest Dermatol 1979;73:150-62.

2. Chang YT, Liu HN, Wang WJ, Lee DD, Tsai SF. A study of cytokeratin profiles in localized cutaneous amyloids. Arch Dermatol Res 2004;296:83-8.

3. Inoue K, Takahashi M, Hamamoto Y, Muto M, Ishihara T. An immunohistochemical study of cytokeratins in skin-limited amyloidosis. Amyloid 2000;7:259-65.

4. Shindo A, Hara H, Terui T. A case of amyopathic dermatomyositis with deposits of amyloid derived from CK5. Skin Research 2005;4:134-7.

5. Orihara T, Yanase S, Furuya T. A case of sclerodermatomyositis with cutaneous amyloidosis. Br J Dermatol 1985;112:213-9.

6. Horiguchi Y, Mitani T, Danno K, et al. Extensive variant of cutaneous amyloidosis: report of a case with electron-microscopic and immunohistochemical studies of the basement membrane zone at sites of amyloid production. Dermatology 1992;185:181-9.

J Rheumatol 2009;36:5; doi:10.3899/jrheum.081148

Personal non-commercial use only. The Journal of Rheumatology Copyright (c) 2009. All rights reserved. 


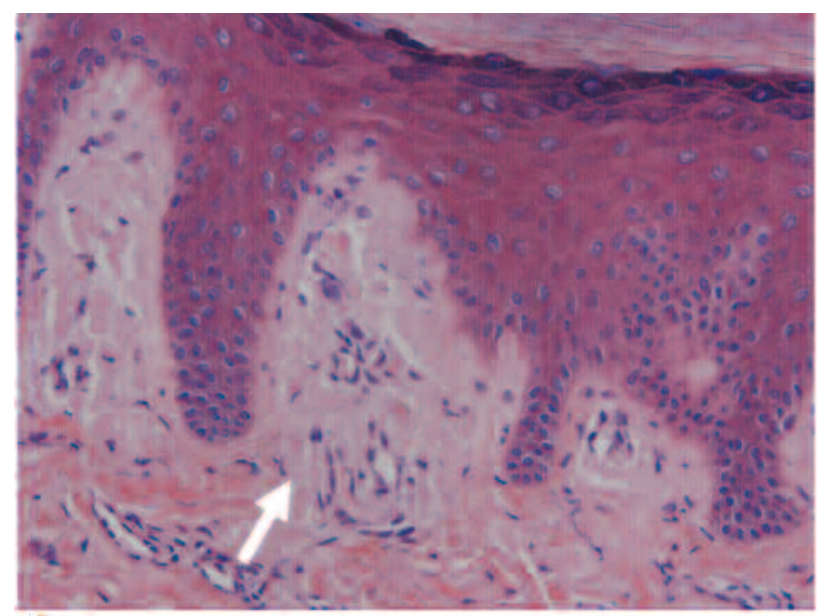

A

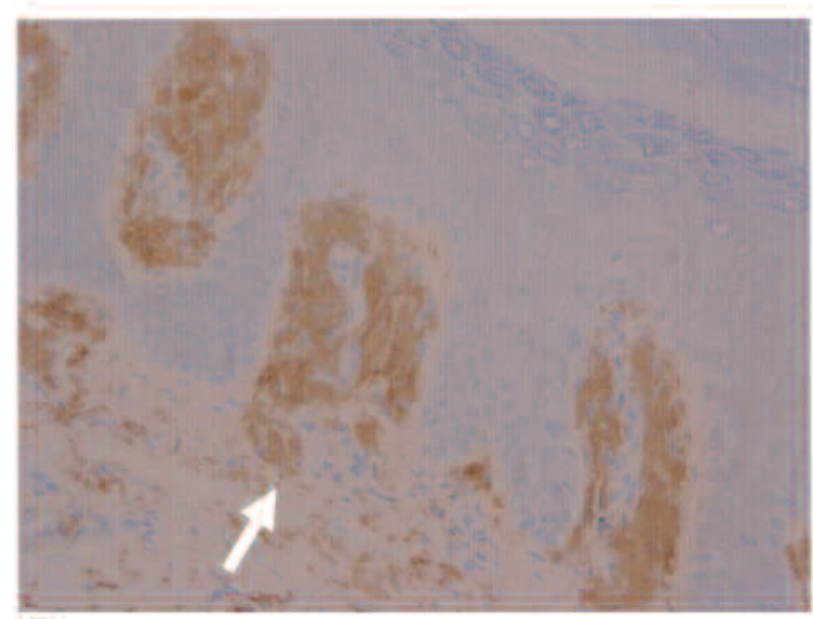

C

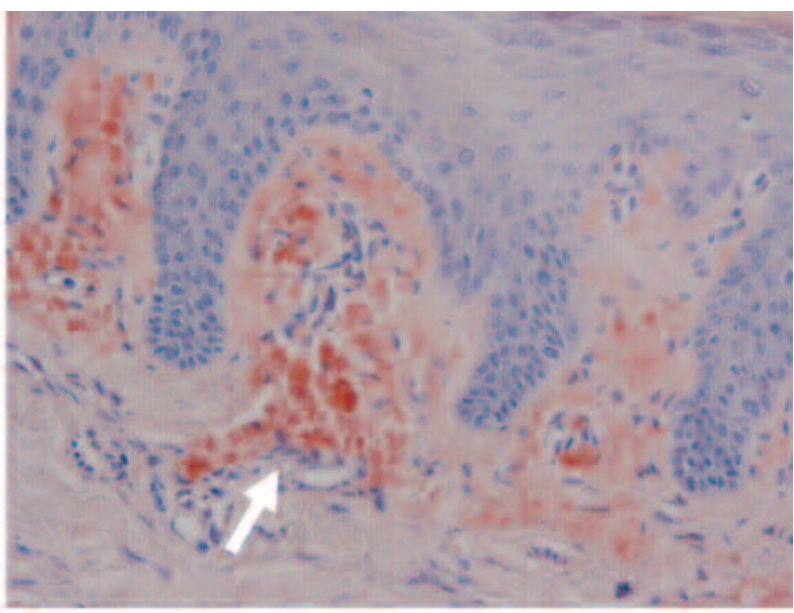

B

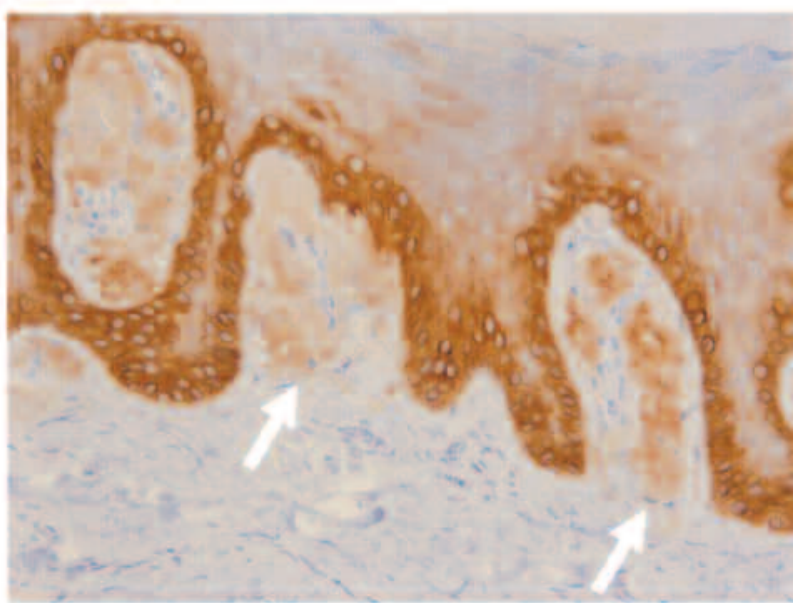

D

Figure 2. Microscopic findings from the skin biopsy. Skin biopsy was performed on the proximal interphalangeal joint of the right fourth finger, which had Gottron's sign. Amyloid deposits were proven in the stratum papillare. A. Skin biopsy of the right fourth finger showed positive H\&E staining. B. Positive staining with Congo red. C. Positive staining with anti-amyloid P antibody. D. Positive staining with anti-cytokeratin 5 antibody. (All original magnification $\times 400$.) 